\title{
La Desertificación del Suelo, Aspectos Y Estrategias de Lucha
}

\section{The Desertification of The Soll, Aspects and CONTROL Strategies}

\author{
${ }^{1}$ Lizeth D. C Molina, ${ }^{2}$ Liliana P. Lozano \\ ${ }^{1}$ Universidad de La Salle, Programa de Ingeniería Ambiental y Sanitaria, Bogotá, D.C. Colombia \\ ${ }^{2}$ Corporación Universidad de la Costa, Barranquilla, Colombia \\ ${ }^{1}$ ldcmolina@unisalle.edu.co \\ ${ }^{2}$ llozano2@cuc.edu.co
}

Recibido: 11/08/2015 • Aprobado: 12/11/2015

\section{Resumen}

La desertificación es el resultado acumulado de un contexto climático difícil y de la utilización inapropiada de la tierra. La recuperación de suelos desertificados comprende todas las actividades que mejoran las tierras de las zonas áridas, semiáridas y subhúmedas secas, con miras a un desarrollo sostenible. Siendo la pérdida de fertilidad del suelo una de las consecuencias más críticas de la desertificación, se hace indispensable el estudio y la adopción de estrategias encaminadas a prevenir y rehabilitar las tierras desertificadas. En el presente artículo, se hace una revisión de estas estrategias, con el objetivo de identificar, reunir, analizar y discutir el conocimiento existente sobre cada una. Se inicia con una revisión de los conceptos, causas y consecuencias ecológicas, económicas y sociales de la desertificación. Seguidamente se presentan las estrategias de prevención y rehabilitación sobre las cuales se han realizado investigaciones, incluyendo estrategias de adaptación y, finalmente, un análisis de las mismas.

Palabras claves: adaptación, desertificación, desarrollo, pobreza, prevención, rehabilitación, tierras.

\section{Abstract}

The desertification of the soil is the cumulative result of difficult climatic circumstances and the inappropriate use of the land. The recovery of desertified land includes all activities that improve the land in arid, semi-arid and dry sub-humid areas, with a view to sustainable development. The loss of soil fertility is one of the most critical consequences of desertification, so it is indispensable the study and the adoption of strategies to prevent and rehabilitate desertified land. In this article, these strategies are reviewed, with the objective to identify, collect, analyze and discuss the existing knowledge about each. It begins with a review of the concepts, causes and ecological, economic and social consequences of desertification. Then, the strategies for prevention and rehabilitation on which research has been conducted, including adaptation strategies, and finally an analysis of them are presented.

Keywords: adaptation, desertification, development, land, prevention, rehabilitation. 


\section{InTRODUCCIÓN}

La desertificación del suelo es una problemática a escala mundial por sus efectos ambientales, económicos y sociales $\mathrm{y}$, principalmente, por su estrecha relación con la pobreza y el hambre considerándose una amenaza para el desarrollo sostenible. El 25\% del área terrestre del planeta está altamente degradada o con altos índices de degradación; cada decenio se pierde unos 120 millones de hectáreas de tierra equivalente a la extensión de Sudáfrica por efecto de la desertificación y la sequía [1]; son 169 los países que han declarado estar afectados por la desertificación [2]. Esta situación es alarmante si se considera que tan solo en América Latina, para el año 2050 , el $50 \%$ de sus tierras podrían estar afectadas por esta problemática [3].

Las cifras de Colombia resultan no menos alentadoras: el $17 \%$ del territorio colombiano se encuentra en proceso de desertificación [4] y el $24 \%$ de sus tierras son susceptibles a esta [5], siendo los departamentos del Atlántico y la Guajira, ubicados en la Región Caribe al norte del país, los más afectados con $93.3 \%$ y $87.5 \%$ de su superficie desertificada [6]. Detener e invertir las tendencias actuales de degradación de la tierra y la desertificación es el próximo paso lógico y rentable para las agendas de desarrollo nacional e internacional [7] en miras de lograr los Objetivos de Desarrollo del Milenio.

La investigación realizada es de tipo descriptiva [8], consistente en una revisión bibliográfica de las estrategias de lucha contra la desertificación encaminadas a la prevención, rehabilitación y adaptación, con el objetivo de proveer bases teóricas que permitan establecer criterios para su implementación. Se escogieron publicaciones realizadas en los últimos seis años, además de aquellas publicaciones que por su reconocimiento en el tema se consideran como básicas. La información obtenida se organizó por subtemas para su revisión y análisis y, finalmente, se estructuró y redactó el resultado o desarrollo de la investigación en el siguiente orden:
- Aspectos generales de la desertificación: definición, causas y consecuencias.

- Estrategias de lucha contra la desertificación: de prevención, de rehabilitación de suelos desertificados y de adaptación a la desertificación.

\section{Aspectos Generales de la Desertificación}

La degradación del suelo se refiere al deterioro de sus características físicas, químicas y/o biológicas que conllevan a la reducción o pérdida de su productividad (biológica y económica) de bienes y servicios, afectando su capacidad de autorregulación y disminuyendo la capacidad actual y futura que tiene el suelo para sustentar la vida humana $[9,10,11,12,13]$. La desertificación se refiere a "la degradación de las tierras de zonas áridas, semiáridas y subhúmedas secas resultante de diversos factores, tales como las variaciones climáticas y las actividades humanas" [14] (esta definición excluye las tierras hiperáridas y hace referencia a la definición internacional establecida por la Convención de las Naciones Unidas de Lucha Contra la Desertificación, aprobada en París, el 17 de junio de 1994, fecha conmemorativa del Día Internacional de Lucha contra la Desertificación).

Este proceso de degradación es exclusivo de las tierras secas [15] y tiene como principales causas las variaciones climáticas y las actividades humanas, factores que no son aislados e independientes, sino sinérgicos. Señalar las variaciones climáticas como una causa independiente y exclusiva de la desertificación, no es justificable debido a que en las regiones áridas los ecosistemas naturales pueden soportar sequías prolongadas prácticamente sin daños, ya que la vegetación y los suelos de éstas regiones se han adaptado a estas condiciones durante miles de años cuando la presión del hombre era aún ligera [16], de manera que, la influencia del clima en los procesos de desertificación se hace 
notoria cuando favorece que las consecuencias derivadas de la acción humana sean mucho más agudas [17] y ejerzan mayor presión sobre los ecosistemas, convirtiéndolos en frágiles para resistir los efectos del clima y la sequía, lo que genera un círculo vicioso de degradación $[18,19,20]$.

De las acciones del hombre se derivan una serie de causas directas, indirectas y subyacentes. Las causas directas por las que el hombre ejerce presión son la deforestación y las inadecuadas prácticas agrícolas; estas dos son condicionadas y fortalecidas por una serie de causas indirectas, alimentadas a su vez por causas subyacentes, las cuales se encuentran identificadas en la Fig. 1 [21], [2], [22], [20], [23, 24, 25, 26, 27] Generalmente, los programas de mitigación y combate de la desertificación se enfocan, equivocadamente solo en las causas directas, dejando en segundo plano las indirectas y las subyacentes, teniendo como resultado poco o nulo éxito en sus acciones [28].

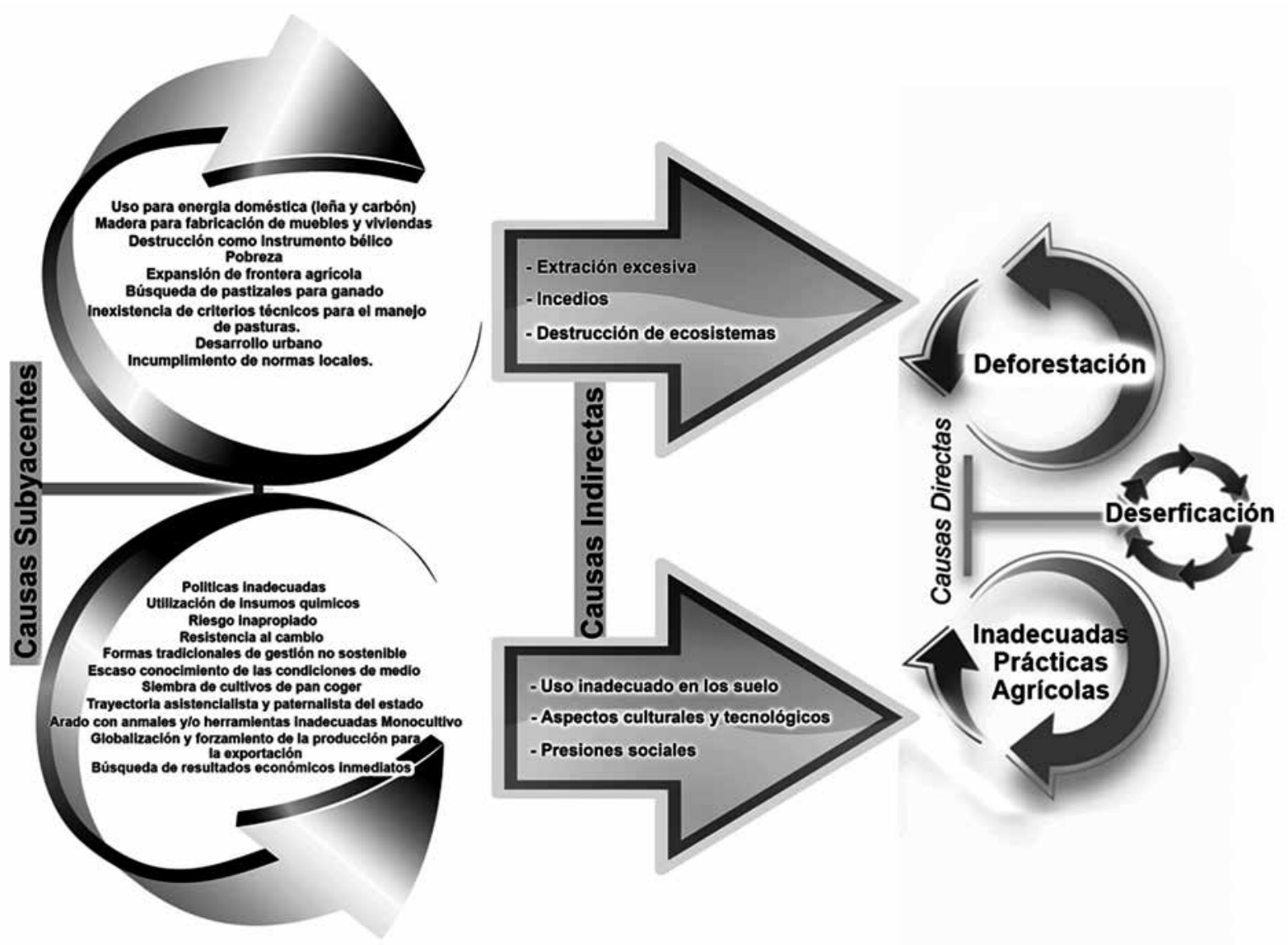

Fig. 1 Causas antrópicas de la desertificación

La principal consecuencia de la desertificación es la pérdida de la capacidad productiva del suelo, desencadenando una serie de factores socioeconómicos como son la pobreza, problemas de salud, desnutrición y mortandad, lo que desemboca incluso en radicalismos, extremismos o guerras por los recursos naturales para la supervivencia, obligando a las comunidades pobres dependientes de la tierra a luchar o emigrar [15], [29, 30]; tan solo como consecuencia de la desertificación, antes de 2045 alrededor de 135 millones de personas podrían desplazarse [31]. En los casos más extremos, el 
hambre y la pobreza se asientan y se convierten tanto en la causa como en la consecuencia de mayor degradación [32]. Sumado a lo anterior, la desertificación también contribuye al cambio climático mundial aumentando el albedo de la superficie terrestre y disminuyendo la tasa actual de evapotranspiración, modificando el equilibrio energético en la superficie y la temperatura del aire contiguo, añadiendo polvo y dióxido de carbono (CO2) a la atmósfera [6], [2].

\section{Estrategias de Lucha Contra la Desertificación}

\section{A. Estrategias para la prevención de la desertifi- cación}

La prevención se logra mediante la adopción de prácticas sostenibles del uso de la tierra. Aquellas que por su efectividad se estudian en este artículo, son:

1. Agricultura de siembra directa - SD: consiste en la implantación de cultivos sin remoción o labranza previa del suelo y con una cobertura permanente de residuos de cosecha anteriores sobre la superficie [33]. Al evitar remover la tierra y mantener una cobertura de rastrojos, se contribuye a mejorar la estructura y estabilidad de los agregados del suelo, incrementar su fertilidad, mantener la humedad y reducir la oxidación de la materia orgánica. Los canales generados por las lombrices y las raíces son más estables y permiten mayor ingreso de agua al perfil; se protege el suelo del impacto de las gotas de lluvia; reduce la velocidad de escurrimiento del agua y la pérdida del suelo en sitios con pendientes; amplía el tiempo de permanencia sobre los residuos para una mejor infiltración, además de obtener un efecto inhibitorio sobre diversos patógenos (plagas, malezas y enfermedades) y de aumentar el rendimiento promedio en la producción de cultivos [34, $35,36,37]$. Para lograr estos efectos, la SD debe complementarse con la rotación de cultivos, de lo contrario, no se obtendrán los resultados de preservación y recuperación del suelo [38, 39].
El $81 \%$ de las tierras de cultivo de Argentina utiliza SD, posicionándose como líder mundial en la adopción de esta tecnología. Con este sistema, se ha logrado allí una ganancia de hasta $100 \mathrm{~mm}$ de agua útil al año con respecto a la labranza convencional, lo que representa un incremento en la producción de $1.700 \mathrm{Kg} / \mathrm{ha}$ de maíz, $1.400 \mathrm{Kg} / \mathrm{ha}$ de sorgo y $800 \mathrm{Kg} / \mathrm{ha}$ de trigo por año [33].

Como aspectos desfavorables de la SD, se considera que al no haber remoción del suelo se proporciona un hábitat adecuado para el aumento en abundancia y diversidad de fauna del suelo (invertebrados, moluscos, anélidos e insectos), algunos de los cuales son perjudiciales para las actividades agrícolas-ganaderas. Sin embargo, estudios realizados señalan que en cultivos de soja en los que se utiliza la SD, no se registran diferencias ni aumentos significativos en la diversidad y en la abundancia de fauna perjudicial con respecto a cultivos en los cuales se utiliza siembra convencional [40].

2. Agricultura forestal o agroforestería: es un sistema de cultivo mixto que busca mantener o imitar algunas de las estrategias que el bosque natural aplica para asegurar nutrientes a toda la cobertura vegetal, con lo cual se pretende lograr un consumo equilibrado de los nutrientes disponibles en el suelo sin que exista el agotamiento de ninguno en particular.

Los sistemas agroforestales combinan el uso de la tierra con especies leñosas (agrosilvicultura), agrícolas (plantación de árboles en granjas ya existentes) y/o animales (silvopastoreo) con el fin de diversificar la producción [21], [41]. El propósito es lograr un sinergismo que conlleve a la protección de los cultivos de la acción del viento gracias a las barreras formadas por los árboles, a la provisión de sombra inicial dada por los cultivos a las plántulas y al aprovechamiento de nutrientes de horizontes inferiores de los suelos a través del sistema radicular de los árboles [42]; los árboles son refugio para las aves, mamíferos y otros organismos los cuales enriquecen el área bajo el dosel con nutrientes mediante sus defecaciones lo que se refleja en la menor 
necesidad de aportes externos de abonos y plaguicidas que otros cultivos sin árboles asociados, además de proteger el suelo contra la erosión eólica e hídrica, mantener la estructura y regular el agua en el suelo [43].

\section{B. Estrategias para la rehabilitación de suelos desertificados}

Sin duda, el aspecto más dañino de la desertificación es la disminución de su capacidad productiva, es decir, su fertilidad, por lo que es necesario recuperar el aporte de materia orgánica y las condiciones ambientales para que los microorganismos edáficos cumplan su papel [21].

Una estrategia, es el uso de enmiendas orgánicas que favorecen la recuperación de la estructura edáfica del suelo, mejoran la composición natural y su contenido de nutrientes, reducen las necesidades hídricas [44], disminuyen la erosión y mejoran la nutrición de las plantas, dando como resultado mayores rendimientos y menor susceptibilidad a los ataques de plaga [45].

La mejora en la composición y las características de abonos se da a través del compostaje, proceso mediante el cual se busca una descomposición biológica de los componentes orgánicos de los materiales desechados que se produce bajo condiciones controladas en las cuales intervienen variados microorganismos (bacterias, hongos y lombrices) que requieren oxígeno, humedad adecuada y sustratos orgánicos heterogéneos en estado sólido [46]. Entre los productos compostados utilizados como enmiendas de suelos se estudian los residuos sólidos, agrícolas y ganaderos:

1. Residuos sólidos: es de destacar el proyecto realizado en San José de Saco, departamento del Atlántico, al norte de Colombia, por la Fundación Manejo Integrado del Medio Ambiente [47], que soluciona una de las principales problemáticas de la localidad como es la generación de 1.500 kilos/día de residuos, los cuales por su inadecuada disposición, ocasionaban deterioro estético, contaminación del suelo y de los cuerpos de agua con los consecuentes problemas de proliferación de vectores y enfermedades en la población. Mediante un acuerdo comunitario se logró dar solución al $90 \%$ de la problemática de disposición de basuras mediante la separación en la fuente en tres categorías: residuos orgánicos, residuos reciclables y materiales peligrosos o no reciclables. Los residuos orgánicos generados se tratan mediante vermicompostaje transformándolos en abono útil para la rehabilitación de suelos desertificados y erosionados de la comunidad, beneficiando familias de escasos recursos al incentivar el cultivo de especies como pepino, acelga, tomate, pimentón, pepino, berenjena, entre otros, y generando ingresos económicos a través de la venta de sus productos mejorando de esta forma su calidad de vida [47].

2. Residuos agrícolas: [48] evaluaron la calidad del compost elaborado a partir de residuos proveniente del proceso de molienda de la caña de azúcar (Saccharum officinarum L.) Los tratamientos fueron: $100 \%$ cachaza ( $\mathrm{T} 1) ; 75 \%$ cachaza y $25 \%$ bagazo (T2); $50 \%$ cachaza y $50 \%$ bagazo (T3); $25 \%$ cachaza y $75 \%$ bagazo (T4) y $100 \%$ bagazo (T5), todos suplementados con $2 \mathrm{~m} 3$ de vinaza. Los resultados obtenidos al final del proceso, 90 días, mostraron que la combinación T3 presentó la mejor calidad con valores de NPK (1,3; 2,5 y 1,0\% respectivamente), valores de carbono orgánico de oxidable de $22.1 \%$ y pH casi neutro (7.1) haciéndolo ideal para ser aplicado en cualquier tipo de suelo, cumpliendo con la Norma Técnica Colombiana NTC-5167 [49].

3. Residuos ganaderos: [50] analizaron el efecto del vermicompostaje producido a partir de estiércol vacuno, sobre algunas propiedades del suelo y la biomasa vegetal. Ellos observaron que al aplicar el vermicompost no solo se aportó nutrientes fundamentales para el crecimiento de las plantas ( $N$ y $\mathrm{P}$ y otros elementos), sino también se mejoró la capacidad de carga de los suelos, lo que provoca un efecto positivo al estimular el crecimiento de estas. Sin embargo, la utilización del vermicompost en proporciones muy altas (mayores del $50 \%$ ) puede tener un efecto negativo en el crecimiento vegetal. 
En suelos desertificados del Sur del departamento del Atlántico, Colombia, se aplicó Eichhorniacrassipes, conocido como Buchón de Agua. Es una planta acuática de rápida proliferación, indicadora de contaminación por presencia excesiva de nutrientes como nitrógeno, fósforo y potasio contribuyendo a los procesos de eutrofización de los cuerpos de agua. Su compostaje permitió utilizarla como mejorador de las condiciones de fertilidad en suelos desertificados logrando así una doble conveniencia: por un lado, la liberación de los espejos de agua con lo que se facilita el drenaje y se lucha contra la eutrofización y, por otro, se logra la producción de materia orgánica para ser aplicada como abono al suelo al momento de la explotación agrícola [26], [51].

\section{Lodos provenientes de planta de tratamiento de aguas residuales (biosólidos)}

Son lodos resultantes del proceso de depuración de aguas residuales que se caracterizan por su alto contenido de materia orgánica y de nutrientes [52]; su composición varía de acuerdo con la procedencia de las aguas residuales por lo que pueden contener organismos patógenos y metales pesados los cuales representarían un peligro para la salud y el medio ambiente. Su disposición final conlleva a problemáticas como la contaminación del suelo, subsuelo y aguas subterráneas, olores desagradables, proliferación de vectores y deterioro estético.

Debido al aumento en su generación, se considera la necesidad de encontrar alternativas sostenibles para los lodos entre las que se encuentran su estabilización mediante tratamientos físico, químico o biológico y la reducción de su potencial contaminante. A este lodo estabilizado, se le conoce como biosólido y su contenido de materia orgánica permite que pueda ser utilizado en el suelo como enmienda orgánica, mejorando sus propiedades biológicas y físicas con lo cual se aumenta el grado de resistencia mecánica de los suelos frente a acciones antrópicas (laboreo) o naturales como el efecto del golpe de la gota de lluvia; mejora la capacidad de intercambio catiónico ya que como coloide contribuye a la absorción de iones, al almacenamiento de elementos nutrientes para las plantas y a la protección física de microorganismos; mejora la porosidad y el drenaje del suelo y la actividad enzimática y microbiológica, lo que avala el uso de lodo residual como fuente potencial de mejoramiento de suelos $[53,54,55]$.

La estabilización de los lodos con altos contenidos de patógenos, coliformes fecales y huevos de helminto mediante composteo y vermicomposteo permite su uso sin ninguna objeción en agricultura, observándose concentraciones de macronutrientes totales $(\mathrm{N}, \mathrm{P} \mathrm{K}$, Ca y Mg), mayores que la de otros abonos orgánicos, y altos contenidos de la fracción extraíble de estos elementos, además de niveles de concentración de metales pesados adecuados o normales, siendo un factor positivo para su uso como abonos $[56,57,58]$.

Entre las limitaciones del uso de lodos residuales como fertilizantes se encuentra que estos pierden progresivamente su contenido de nitrógeno y fósforo, según se reduce su contenido de agua lo que da como resultado proporciones de $\mathrm{N}, \mathrm{P}$ y K, con una concentración alrededor del 3\%. Los lodos tienen un valor fertilizante más alto cuando se utilizan en la fase líquida. Otra limitación es el hecho de que la capacidad de adsorción de estos hace que los lodos acumulen metales pesados en los mismos. Como los metales pesados permanecen junto a los sólidos de los lodos, su concentración aumenta según se reduce progresivamente el contenido de agua [59].

\section{Lodos provenientes de industrias del papel}

En el caso de lodos provenientes de la industria de papel se ha planteado que su uso estaría orientado básicamente a fungir como enmienda, dado su bajo contenido de nutrientes [60]. Sin embargo, ensayos en campo han mostrado incrementos en los rendimientos para algunos cultivos, crecimiento del carbono microbiano y de la actividad de la enzima deshidrogenada, resultando un buen indicador de la actividad microbiana [61, 62].

\section{E. Estrategias de adaptación a la desertificación}

Cuando la problemática de la desertificación es irreversible, o cuando los costos para su prevención 
y rehabilitación superan las ganancias de producción de los terrenos, se deben considerar alternativas de adaptación. La alternativa más conocida es el riego por goteo, que se viene implementando en países desarrollados, pero con limitaciones de recursos naturales como Israel, pionero en esta tecnología. Consiste en el suministro uniforme de agua a través de tuberías con perforaciones que controlan el caudal de agua que recibe la planta. El riego por goteo, como estrategia de adaptación a la desertificación, permite que los suelos desertificados se aprovechen con fines agrícolas al ser utilizados únicamente como soporte, puesto que el sustrato necesario para el desarrollo de las plantas es suministrado por medio de este sistema.

Esta tecnología se justifica a pequeña escala y en escenarios con limitaciones de suelos agrícolas, y de recursos hídricos y energéticos. Su utilización ofrece perspectivas favorables de contribuir a la producción hortícola y representa una alternativa promisoria para ser introducida en los programas nacionales de la agricultura [63]. No solo permite un uso de los suelos desertificados, sino que mejora el aprovechamiento del agua en áreas con grandes limitaciones para acceder a este recurso.

Investigaciones realizadas afirman que con el riego por goteo se logra un ahorro de agua entre el $30 \mathrm{y} \mathrm{el}$ $50 \%$ con respecto al riego por aspersión, por inundación y/o continuo, debido a que la aplicación de agua es de bajo volumen en comparación con dichos sistemas de riego, lo que permite conservar el agua, incrementar significativamente la calidad y producción de los cultivos y aumentar el área de riego. Entre las ventajas adicionales se encuentra la aplicación de agroquímicos por medio de inyección (fertirriego) y, por ende, el ahorro en mano de obra y un mejor aprovechamiento en los nutrimentos por el cultivo, aspecto que se destaca en relación con el uso de suelos desertificados para la agricultura [64], [63], [65, 66].

\section{ANÁLISIS}

Las estrategias presentadas en esta revisión, atienden a una situación en particular del grado de desertificación: prevención, rehabilitación y adaptación; sin embargo, estas estrategias no serán del todo efectivas si no van acompañadas de acciones que conduzcan a una gestión sostenible de las tierras secas tales como la declaración de áreas protegidas; el mantenimiento de la funcionalidad y sostenibilidad de los ecosistemas, en especial los estratégicos; los programas de conservación de la biodiversidad en tierras secas y el acompañamiento al sector agrícola como sector clave en la producción de alimentos para la población.

Haciendo un análisis de cada una de las estrategias presentadas, desde el punto de vista de los autores se puede señalar lo indicado en la Tabla 1:

TABLA I.

Análisis de las Estrategias de lucha Contra la Desertificación

\begin{tabular}{|c|c|c|c|c|c|}
\hline TIPO & ESTRATEGIA & $\begin{array}{l}\text { REQUERI- } \\
\text { MIENTOS }\end{array}$ & VENTAJAS & DESVENTAJAS & ANÁLISIS \\
\hline \multirow[b]{2}{*}{ Prevención } & $\begin{array}{l}\text { Agricultura } \\
\text { de siembra } \\
\text { directa (SD) }\end{array}$ & $\begin{array}{l}\text { Rotación } \\
\text { de cultivos. }\end{array}$ & $\begin{array}{l}\text { - Mejora la estructura } \\
\text { del suelo. } \\
\text { - Conserva la humedad } \\
\text {-y biporosidad del suelo. } \\
\text { - Aumenta la fertilidad. } \\
\text { - Reduce el escurrimiento } \\
\text { y la erosión. }\end{array}$ & $\begin{array}{l}\text { Posible aumento de mi- } \\
\text { crofauna perjudicial para } \\
\text { los cultivos }\end{array}$ & \multirow{2}{*}{$\begin{array}{l}\text { La prevención es la opción } \\
\text { más económica y sostenible } \\
\text { para luchar contra la deser- } \\
\text { tificación. Estas estrategias } \\
\text { requieren de políticas nacio- } \\
\text { nales tendientes a disminuir } \\
\text { los riesgos de desertifica- } \\
\text { ción en tierras susceptibles. } \\
\text { Dichas políticas deben pro- } \\
\text { curar la destinación de es- } \\
\text { fuerzos y recursos para la } \\
\text { investigación, protección de } \\
\text { los suelos, y la concientiza- } \\
\text { ción y capacitación de las } \\
\text { comunidades en técnicas } \\
\text { agrícolas sostenibles. }\end{array}$} \\
\hline & Agroforestería & $\begin{array}{l}\text { Especies con } \\
\text { requerimientos } \\
\text { nutricionales } \\
\text { diferentes. }\end{array}$ & $\begin{array}{l}\text { - Se reduce el agotamiento } \\
\text { de nutrientes del suelo. } \\
\text { - Menor uso de abonos } \\
\text { y plaguicidas. } \\
\text { - Potencialización del uso } \\
\text { de la tierra. } \\
\text { - Regulación del agua } \\
\text { en el suelo. } \\
\text { - Protección contra la erosión. }\end{array}$ & $\begin{array}{l}\text { - La combinación de } \\
\text { agrosilvicultura y } \\
\text { silvopastoreo, puede } \\
\text { ocasionar daños en los } \\
\text { cultivos al ser deterio- } \\
\text { rados por el paso de } \\
\text { animales. } \\
\text { - Las producción de } \\
\text { cultivos se ve limitada } \\
\text { por las extensiones de } \\
\text { tierra disponible. }\end{array}$ & \\
\hline
\end{tabular}




\begin{tabular}{|c|c|c|c|c|c|}
\hline TIPO & ESTRATEGIA & $\begin{array}{l}\text { REQUERI- } \\
\text { MIENTOS }\end{array}$ & VENTAJAS & DESVENTAJAS & ANÁLISIS \\
\hline \multirow{3}{*}{$\begin{array}{l}\text { Rehabilita- } \\
\text { ción }\end{array}$} & $\begin{array}{l}\text { Compostaje } \\
\text { de residuos } \\
\text { sólidos, } \\
\text { agrícolas } \\
\text { y ganaderos }\end{array}$ & $\begin{array}{l}\text { - Separación de } \\
\text { residuos en la } \\
\text { fuente genera- } \\
\text { dora. } \\
\text { - Cercanía de la } \\
\text { fuente genera- } \\
\text { dora al sitio de } \\
\text { aplicación. } \\
\text { - Infraestructura } \\
\text { disponible y } \\
\text { control de fac- } \\
\text { tores y varia- } \\
\text { bles durante } \\
\text { el proceso de } \\
\text { compostaje. }\end{array}$ & $\begin{array}{l}\text {-Aprovechamiento de } \\
\text { residuos, disminuyendo los } \\
\text { problemas de contaminación } \\
\text { asociados a su disposición. } \\
\text { - Producto con alto contenido } \\
\text { de materia orgánica que } \\
\text { mejora la estructura del } \\
\text { suelo y aumenta su fertilidad. }\end{array}$ & $\begin{array}{l}\text { La aplicación de propor- } \\
\text { ciones muy altas puede } \\
\text { tener un efecto negativo } \\
\text { en el crecimiento de las } \\
\text { plantas. }\end{array}$ & \multirow{3}{*}{$\begin{array}{l}\text { El uso de enmiendas y/o } \\
\text { abonos es la alternativa } \\
\text { ideal para aportar los nu- } \\
\text { trientes perdidos en suelos } \\
\text { desertificados. Los resi- } \\
\text { duos compostados es una } \\
\text { alternativa para la solución } \\
\text { de diversas problemáticas } \\
\text { ambientales: } \\
\text { - Aprovechamiento de } \\
\text { residuos cuya generación } \\
\text { va en aumento. } \\
\text { - Contaminación de suelos } \\
\text { y aguas asociada a su } \\
\text { comúnmente mala dispo- } \\
\text { sición. } \\
\text { - Reducción de áreas } \\
\text { destinadas a su disposi- } \\
\text { ción final. } \\
\text { Esta alternativa en auge, } \\
\text { amplia cada vez más la } \\
\text { posibilidad de aprovechar } \\
\text { residuos de diversas } \\
\text { características, composi- } \\
\text { ción y origen, por lo que } \\
\text { la investigación en esta } \\
\text { área se encuentra en } \\
\text { crecimiento. } \\
\text { Sin embargo, esta } \\
\text { estrategia presenta difi- } \\
\text { cultades al momento de } \\
\text { su aplicación a escalas } \\
\text { reales por factores como } \\
\text { es el traslado desde el } \\
\text { sitio de generación de } \\
\text { los residuos hasta el } \\
\text { lugar de su estabilización } \\
\text { y posteriormente a las } \\
\text { áreas afectadas. } \\
\end{array}$} \\
\hline & $\begin{array}{l}\text { Compostaje } \\
\text { de Biosólidos }\end{array}$ & $\begin{array}{l}\text { - Su valor } \\
\text { fertilizante } \\
\text { está sujeto al } \\
\text { mantenimien- } \\
\text { to de la fase } \\
\text { líquida en los } \\
\text { lodos. } \\
\text { - Requiere } \\
\text { períodos de } \\
\text { estabilización } \\
\text { largos para la } \\
\text { eliminación de } \\
\text { organismos } \\
\text { patógenos } \\
\text { y reducir su } \\
\text { potencial con- } \\
\text { taminante. }\end{array}$ & $\begin{array}{l}\text { - Mejora de propiedades } \\
\text { biológicas y físicas del suelo } \\
\text { (CIC, almacenamiento de } \\
\text { nutrientes, incremento de } \\
\text { la porosidad y drenaje del } \\
\text { suelo, mejora la actividad } \\
\text { enzimática y microbiológica). } \\
\text { - Proporciona al suelo re- } \\
\text { sistencia frente a acciones } \\
\text { antrópicas y naturales. } \\
\text { Contienen concentraciones } \\
\text { de macronutrientes totales } \\
\text { (N, P K, Ca y Mg) y altos } \\
\text { contenidos de la fracción } \\
\text { extraíble de estos elemen- } \\
\text { tos adecuados para su uso } \\
\text { como abono. }\end{array}$ & $\begin{array}{l}\text { - Contenido de patógenos } \\
\text { y/o metales pesados } \\
\text { que pueden contaminar } \\
\text { suelos y acuíferos. } \\
\text { - Pérdida progresiva de } \\
\text { nutrientes y aumento de } \\
\text { la concentración de me- } \\
\text { tales pesados, conforme } \\
\text { se reduce su contenido } \\
\text { de agua. }\end{array}$ & \\
\hline & $\begin{array}{l}\text { Compostaje } \\
\text { de lodos } \\
\text { papeleros }\end{array}$ & $\begin{array}{l}\text { Requiere la } \\
\text { utilización de } \\
\text { otros pro- } \\
\text { ductos para } \\
\text { mejorar su } \\
\text { bajo contenido } \\
\text { de nutrientes } \\
\text { (residuos } \\
\text { ganaderos, } \\
\text { orgánicos y } \\
\text { agrícolas) }\end{array}$ & $\begin{array}{l}\text { - Buen indicador de actividad } \\
\text { microbiana. } \\
\text { - Mejora propiedades físicas } \\
\text { del suelo como } \\
\text { la porosidad. }\end{array}$ & $\begin{array}{l}\text { - Bajo contenido de } \\
\text { nutrientes. } \\
\text { - En altos porcentajes } \\
\text { puede inhibir el creci- } \\
\text { miento de las plantas y } \\
\text { aumentar las condicio- } \\
\text { nes de anoxia en suelos } \\
\text { por lo que se requiere } \\
\text { la mezcla con otras } \\
\text { enmiendas. }\end{array}$ & \\
\hline Adaptación & $\begin{array}{l}\text { Riego } \\
\text { por goteo } \\
\text { o Fertiriego }\end{array}$ & $\begin{array}{l}\text { - Entrenamiento } \\
\text { del personal } \\
\text { en el uso de la } \\
\text { técnica. } \\
\text { Conocimiento } \\
\text { de la deman- } \\
\text { da de agua y } \\
\text { requerimiento } \\
\text { de los culti- } \\
\text { vos. } \\
\text { - Si bien reduce } \\
\text { el consumo } \\
\text { de agua, } \\
\text { requiere de } \\
\text { la disponibi- } \\
\text { lidad de este } \\
\text { recurso para } \\
\text { su funciona- } \\
\text { miento }\end{array}$ & $\begin{array}{l}\text { - Uso de suelos desertificados } \\
\text { como soporte de cultivos. } \\
\text { - Reduce la cantidad de agua } \\
\text { empleada para riego. } \\
\text { - Suministro directo de } \\
\text { nutrientes requeridos a la } \\
\text { planta. }\end{array}$ & $\begin{array}{l}\text { Costos iniciales de } \\
\text { inversión, sin embargo } \\
\text { la relación costos/be- } \\
\text { neficios es satisfactoria } \\
\text { al considerar el uso de } \\
\text { suelos desertificados y } \\
\text { el uso eficiente del agua } \\
\text { en zonas con limitación } \\
\text { de acceso y disponibili- } \\
\text { dad del recurso. }\end{array}$ & $\begin{array}{l}\text { Aunque esta tecnología } \\
\text { pionera de países con } \\
\text { problemas de desertifica- } \\
\text { ción irreversibles, puede } \\
\text { considerarse también una } \\
\text { estrategia de prevención. } \\
\text { La organización del sector } \\
\text { agrícola es fundamental } \\
\text { para el aprovechamiento } \\
\text { de esta tecnología, ya que } \\
\text { por sus costos, pequeños } \\
\text { agricultores dificultosa- } \\
\text { mente podrían acceder } \\
\text { a ella. }\end{array}$ \\
\hline
\end{tabular}




\section{Conclusión}

Mediante la revisión realizada se concluye que, si bien la restauración de los suelos desertificados debe conducir a la recuperación de su fertilidad, son realmente las medidas de prevención y adecuado uso de los mismos en las que se deben centrar los esfuerzos. La prevención requiere de cultura, educación e incentivos, dado que en muchos casos los programas de mitigación y combate de la desertificación se enfocan, equivocadamente, solo a las causas directas dejando en segundo plano las causas indirectas y subyacentes teniendo como resultado poco o nulo éxito en sus acciones.

Las técnicas de compostaje de material orgánico ofrecen una amplia gama de posibilidades con efectos positivos sobre la estructura del suelo, permitiéndole a este recuperar características importantes para su productividad. De estos materiales orgánicos, el compostaje de lodos es una alternativa que debe considerarse, puesto que su aplicación tiene un doble efecto ambiental positivo: acondiciona y mejora el suelo, y se reduce su volumen destinado a su disposición final. Se recomienda la profundización de los beneficios de la aplicación de lodos procedentes de diferentes fuentes en el suelo, mediante la realización de investigaciones que puedan establecer parámetros para su provecho y aplicación.

\section{REFERENCIAS}

[1] FAO. "The state of the world's land and water resources for food and agriculture (SOLAW) Managing systems at risk", 2011.p113.

[2] CNULD. "Adaptación y resiliencia basada en la tierra impulsadas por la naturaleza. Convención de las Naciones Unidas de lucha contra la Desertificación", Alemania, p2 2014a.

[3] IFAD. (2010, Agosto). Desertification. [Online]. Available: http://www.wmo.int/youth/sites/default/files/field/media/library/idad-desertification.pdf

[4] MADS-IDEAM. (2012). Problemática de la degradación de suelos y tierra en Colombia. Taller Nacional realizado el viernes 30 de noviembre de 2012. [Online] Available: https:// www.siac.gov.co/documentos/DOC_Portal/DOC_Suelo/20121210 Problematica degradaci\%C3\%B3n Suelos Tierras_Colombia_MADS.pdf
[5] SIAC. (2015). Web site del Sistema de Información Ambiental de Colombia. [Online]. Available: https://www.siac.gov.co/ contenido/contenido.aspx?cat $\mid \mathrm{D}=893 \&$ con $I \mathrm{D}=1432$

[6] PNUD. "Estado de avance de los Objetivos de Desarrollo del Milenio. Programa de las Naciones Unidas para el Desarrollo". Guajira. Colombia. 2012.

[7] CNULD. "La Tierra en cifras, los medios de subsistencia en su punto de inflexión". Convención de las Naciones Unidas de lucha contra la Desertificación, Alemania. 2014b.

[8] A. Merino. "Cómo escribir documentos científicos (parte 3)". Artículo de revisión. Revista Salud en Tabasco, Vol. 17, núm. 1-2, pp 34-40. México. 2011.

[9] D. De la Rosa. "Evaluación agro-ecológica de suelos: para un desarrollo rural sostenible." España. Mundi - Prensa. 2008.

[10] FAO. "Metodología provisional para la evaluación de la degradación de los suelos." FAO/PNUMA.FAO4. Roma. 1980.

[11] OMM. "El clima y la degradación de tierras". Organización Meteorológica Mundial. No. 989. 2006.

[12] M. Espinosa, E. Andrade. P. Rivera y A. Romero. "Degradación de suelos por actividades antrópicas en el norte de Tamaulipas, México". Revista Papeles de Geografía. Número 53-54, pp 77-88. Universidad de Murcia, España. 2011.

[13] CNULD, "Neutralidad en la degradación de la tierra. La resiliencia a escala local, nacional y regional". Convención de las Naciones Unidas de lucha contra la Desertificación, Alemania, p24. 2014c

[14] ONU. Programa 21. [Online]. Available: http://www.un.org/ spanish/esa/sustdev/agenda21/agenda21spchapter11.htm

[15] CNULD. "La desertificación. Esa invisible línea de frente". Convención de las Naciones Unidas de lucha contra la Desertificación, Alemania, p18. 2014d.

[16] D. Granados, M. Hernández, A. Vázquez y P. Ruíz, "Los procesos de desertificación y las regiones áridas", Revista Chapingo. Serie Ciencias Forestales y del Ambiente 19(1). pp 45-66. 2013.

[17] C. Morales, S. Parada, y S. Pobreza," Desertificación y degradación de los recursos naturales". Comisión Económica para América Latina y el Caribe (CEPAL) Santiago de Chile, p273. 2005.

[18] I. Soares y B. De Souza, "Solos e desertificação no sertão paraibano". Revista Caderno do Logepa 6(2). pp101114. 2011.

[19] A. Pereira. y M. Soares, "Degradação ambiental e desertificação no semiárido mineiro: um estudo sobre o município de Espinosa (MG).' Revista Geográfica de América Central, Número Especial EGAL. pp 1-16. 2011.

[20] F. López. (2012, Mayo). Desertificación, un riesgo ambiental global de graves consecuencias. Cuaternario y Geomorfología, Norteamérica, 20. [Online]. Available: http://recyt.fecyt.es/index.php/CUGEO/article/view/16991/10406

[21] C. Peña y G. Cardona. "Dinámica de los suelos amazónicos. Procesos de degradación y alternativas para su recuperación". Instituto Sinchi, Bogotá, 2010, p115. 
[22] L. Torres, "Claroscuros del desarrollo sustentable y la lucha contra la desertificación: las racionalidades económicas en el ojo de la tormenta. Estudio de caso con productores caprinos de tierras secas (Mendoza, Argentina).' Revista Mundo Agrario, Vol. 11 No. 21. 2014.

[23] K. Mora. (2011). Prácticas agrícolas coloniales y degradación del suelo: el caso de Saquencipá. Revista Geográfica de América Central, Norteamérica, 2. [Online]. Available: http://www.revistas.una.ac.cr/index.php/geografica/article/ view/2418

[24] CNULD, "El pastoreo femenino. La conservación de los conocimientos tradicionales. Respuestas a los nuevos desafíos". Secretaría de la Convención de las Naciones Unidas de lucha contra la Desertificación, Alemania, 2007, p45.

[25] S. Rigotti, "El conflicto del campo. Matrices culturales e identificaciones políticas." Revista Mundo Agrario, 15(29). 2014.

[26] Fondo de Asistencia del Sector Privado (FASEP). (2011) Gestión de las aguas y del suelo del Embalse del Guájaro. Informe sobre manejo agrícola y conservación de suelos. [Online]. Avalaible: http://www.aigos.com.co/guajaro/es/fscommand/informeSUME.pdf.

[27] ESC. "Plan de manejo ambiental del distrito de manejo integrado - DMI- Luriza, Usiacurí - Atlántico," Fundación Ecosistemas Secos -ESC. Colombia. 2011.

[28] J. Pulido y G. Bocco, “¿Cómo se evalúa la degradación de tierras? Panorama global y local.' Revista Interciencia. Volumen 36 No. 2. pp 96-103. 2011.

[29] E. Andrade, M. Espinosa y A. Romero, "Acciones de lucha contra la desertificación en ambientes semiáridos en el noroeste de Tamaulipas, México,' Revista Papeles de Geografía, núm 49—50, 2009, pp15-26.

[30] M. Jaramillo. "Desertification and Infant Mortality in Colombia." En el marco de The 5th International Conference on Drylands, Deserts and Desertification. Blaustein Institutes for Desert Research Sede Boqer Campus of Ben-Gurion University, Israel. 2014.

[31] Global Humanitarian Forum (GHF). (2009). Human Impact Report - Climate Change. The Anatomy of A Silen t Crisis. GHF, Geneva. [Online]. Available: http://www.ghf-ge.org/ human-impact-report.pdf

[32] CNULD. "Las tierras y los suelos en el contexto de una economía verde para lograr el desarrollo sostenible, la seguridad alimentaria y la pobreza". La presentación de la Secretaría de la CNULD para el proceso de la conferencia Río+20. Alemania. 2011.

[33] INTA. (2011, Febrero). Siembra Directa. Actualización Técnica No. 58. Instituto Nacional de Tecnología Agropecuaria. Argentina. [Online]. Available: http://inta.gob.ar/documentos/siembra-directa/at_multi_download/file/Siembra\%20 Directa\%202011.pdf

[34] M. Báez y J. Aguirre, "Efecto de la labranza de conservación sobre las propiedades del suelo". Tierra Latinoamericana, vol. 29, núm 2, 2011, pp 113-121.

[35] A. Navarro y otros. "Propiedades físicas y químicas del suelo bajo labranza de conservación y su relación con el rendimiento de tres cultivos". Revista Mexicana de Ciencias Agrícolas. Núm 4, 2012, pp 690-697.
[36] A. Báez-Pérez y otros, "Implementación de la siembra directa para producción de cebada maltera en el estado de Guanajato"' Revista Mexicana de Ciencias Agrícolas. Vol. 3, núm 8. 2012, pp 1509-1519.

[37] R. Crespo y otros, "Efecto de la labranza y la cobertura vegetal sobre el escurrimiento y la pérdida de suelo en la Región Central de la provincia de Buenos Aires". Revista de la Facultad de Ciencias Agrarias. Tomo 42 No. 1, 2010, pp 93-106.

[38] F. Castilla. "Siembra directa: la elegida para conservar el suelo." Revista de Investigaciones Agropecuarias. 013. 2013. Vol. 39 No. 2, 2013.

[39] P. Campitelli y otros, "Selección de indicadores de calidad de suelo para determinar los efectos del uso y prácticas agrícolas en un área piloto de la región central de Córdoba". Revista Ciencia del Suelo. 28(2), 2010, pp 223-231.

[40] D. Scuffi y otros, "¿La práctica de la siembra directa en cultivos de soja favorece las poblaciones de acridios (Orthoptera: Acrididae) en el partido de Benito Juárez?", Revista de la Sociedad Entomológica Argentina. Vol. 71, núm. 3-1, 2012, pp 203-213.

[41] D. Cabrera y otros, (2011) Establecimiento de Sistemas Agroforestales. Instituto nacional de Investigaciones Forestales, Agrícolas y Pecuarias (INIFAP). Primera edición. México. [Online]. Avalaible:http://www.academia. edu/6286266/MANUAL_SISTEMAS_AGROFORESTALES_PDF?login=\&email_was_taken=true

[42] T. May, "Sistemas agroforestales de colonos como alternativas de uso ecológicamente sustentable en el oeste de Pará, Brasil. Adopción y propuestas para su desarrollo", Ambiente y Desarrollo. Vol. 17(32), 2013, pp 67-78.

[43] B. Moran, A. Herrera, y K. López, "Evaluación socioeconómica y ambiental de tres tipos de sistemas agroforestales en el Trópico Seco Nigaragüense", Revista Científica de FAREM-Estelí. Medio ambiente, tecnología y desarrollo humano. No 11, año 3, 2014.

[44] F. Ramos y otros, "Efecto de abonos orgánicos en el rendimiento del cultivo de chile ancho (Capsicum annuum L.), y sobre las características químicas del suelo de la parcela experimental,' Revista Investigación y Ciencia. 19(51), 2011, pp 3-9.

[45] M. Sánchez y M. Viera. "Producción y utilización de abonos orgánicos para mejorar la composición de los suelos como estrategia para obtener rubros alimenticios de mejor calidad nutritiva", Universidad Politécnica Territorial de Alto Apure Pedro Camejo Núcleo Elorza, 2012.

[46] D. Sánchez, "Estudio de factibilidad para la creación de la planta de abonos orgánicos de Colombia." Proyecto de emprendimiento para optar por el título de Administrador del Medio Ambiente y de los Recursos Naturales. Universidad Autónoma de Occidente, 2013.

[47] Fundación Manejo Integrado del Medio Ambiente (MIMA). (2014). Experiencia en San José del Saco, Atlántico. [Online]. Available: https://www.youtube.com/ watch? $\mathrm{t}=469 \& \mathrm{v}=$ aqcu5yuPTsk

[48] A. Bohórquez, Y. Puentes y J. Menjivar. "Evaluación de la calidad del compost producido a partir de subproductos agroindustriales de caña de azúcar", Corpoica Cienc. Tecnol. Agropecu. Manejo y Conservación de Suelos y Aguas. (1), 2014, pp 73-81. 
[49] NTC-5167. Productos para la industria agrícola. Productos orgánicos usados como abonos o fertilizantes y enmiendas o acondicionadores de suelo. (2004-31-05).

[50] L. Durán y C. Henríquez, "El vermicompost: su efecto en algunas propiedades del suelo y la respuesta de la planta, Revista Agronomía Mesoamericana. 21(1), 2009, pp 85-93.

[51] I. Caro, Z. Romero y R. Lora, "Producción de abonos orgánicos con la utilización de Elodea (Egeria densa) presente en la laguna de Fúquene", Revista U.D.C.A Actualidad \& Divulgación Científica. 12(1), 2009, pp 91-100.

[52] F. Colomer y otros, "Opción de valorización de lodos de distintas estaciones depuradoras de aguas residuales", Ingeniería. Vol. 14 No.3, 2010, pp 177-190.

[53] M. Valderrama, "Factibilidad de aprovechamiento de los lodos residuales de la PTAR el Municipio de Chinavita (Boyacá)," Trabajo de investigación presentado como requisito para optar por el título de Magister en Desarrollo Sostenible y Medio Ambiente. Universidad de Manizales. Colombia, 2013.

[54] S. Builes, "Tratamiento y adecuada disposición de lodos domésticos e Industriales", Trabajo de monografía para optar por el Título de Tecnólogo Químico. Facultad de Tecnologías. Universidad Tecnológica de Pereira, 2010.

[55] L. Ruesga, "Efecto de la aplicación de lodo durante el cultivo de cebollín (Allium fistulosum L) en Barbacoas,' Trabajo de grado para optar por el título de Magíster Scientiarum en Ingeniería Ambiental. La Universidad del Zulia, Venezuela, 2010.

[56] M. Vicencio, M. Pérez, E. Medina y M. Martínez, "Producción de composta y vermicomposta a partir de lodos de la planta de tratamiento de aguas residuales de un rastro, Revista Internacional de Contaminación Ambiental. UNAM, Vol. 27, núm 3, 2011, pp 263-270.

[57] J. Gilsanz, C. Leoni, F. Schelotto y A Acuña, "Uso potencial de los lodos urbanos en la producción agrícola", Agrociencia Uruguay. Volumen 17:2, 2013, pp 1-10.
[58] E. Robledo y otros, "Sales solubles y metales pesados en suelos tratados con biosólidos", Revista Chapingo Serie Ciencias Forestales y del Ambiente. 16(2), 2010, pp 241-251

[59] M. García, "Rehabilitación de un suelo con bajo perfil de nutrientes aplicando biosólidos como fertilizante", Tesis. Instituto Politécnico Nacional. México, 2011.

[60] C. J. Beauchamp, H. Charest and A. Gosselin, "Examination of environmental quality of raw and composting de-inking paper sludge. Chemosphere", 46(6) 2002, pp 887-895.

[61] K. Bellamy et al, "Paper Sludge Utilization in Agriculture and Container Nursery Culture." J. Environ. Qual. 24, 1995, pp 1074-1082.

[62] A. García y C. Rivero, "Efecto de la aplicación de lodos papeleros sobre los contenidos de carbono microbiano y la actividad de deshidrogenasa en suelos agrícolas,' Revista Venesuelos. 18, 2011, pp 29-35.

[63] M. Rodríguez y otros, "Mejoramiento de riego por surcos, continuo e intermitente, en suelo ferralítico rojo lixiviado en el sistema productivo Banao, Revista Ciencias Técnicas Agropecuarias, Vol. 23, No1, 2014, pp 56-59.

[64] J. Montemayor y otros, "Uso del agua en la alfalfa (Meedicago sativa) con riego por goteo subsuperficial," Revista Mexicana de Ciencias Pecuarias, Vol. 1(2), 2010, pp 145-156.

[65] M. Rodríguez y T. López, "Comportamiento de la zona radical activa del banano en un Ferrasol bajo riego por goteo superficial y subsuperficial," Revista Ciencias Técnicas Agropecuarias, Vol. 23, No. 3, 2014, pp 5-10.

[66] M. Lang y otros, "Ensayo comparativo de rendimiento de cinco cultivares bienales de zanahoria (Daucus carota L.) en la región semiárida pampeana, bajo riego por goteo", Semiárida Revista de la Facultad de Agronomía UNL Pam Vol. 24(1), 2014, pp 49-54. 
